\title{
Neue Wege in der Thorax-Onkologie
}

\section{New Diagnostic and Therapeutic Strategies in Thoracic Oncology}

Autoren

Institute
N. Reinmuth ${ }^{1}$, C. Grohé ${ }^{2}$

${ }^{1}$ LungenClinic Grosshansdorf, Abteilung für Onkologie

${ }^{2}$ Evangelische Lungenklinik Berlin, Klinik für Pneumologie
Bibliografie

DOI http://dx.doi.org/

10.1055/s-0032-1326362

Pneumologie 2013; 67: 197

(c) Georg Thieme Verlag KG

Stuttgart · New York

ISSN 0934-8387

\section{Korrespondenzadresse \\ PD Dr. Niels Reinmuth}

Oberarzt

LungenClinic Grosshansdorf

$\mathrm{GmbH}$

Wöhrendamm 80

22927 Großhansdorf

n.reinmuth@lungenclinic.de

\section{Prof. Dr. med. Christian Grohé}

Chefarzt der Pneumologischen Klinik

Evangelische Lungenklinik Berlin Krankenhausbetriebs gGmbH Lindenberger Weg 27

13125 Berlin

christian.grohe@elk-berlin.de
Die diagnostischen und therapeutischen Ansätze in der Thorax-Onkologie erleben zurzeit eine Vielzahl von richtungweisenden Änderungen. Durch ein zunehmendes und tieferes Verständnis molekularer Veränderungen von Tumorzellen konnten neue Zielstrukturen identifiziert werden, die individuelle Therapien ermöglichen. Für die Behandlung des Lungenkarzinoms hat sich das therapeutische Spektrum erweitert, aber auch die Diagnostik wird zunehmend komplexer. Mit Hilfe teilweise sehr unterschiedlicher molekularbiologischer Methoden sollen aktuell und zukünftig vermehrt verschiedene genetische Zielstrukturen detektiert werden. Dieser Entwicklung Rechnung tragend, haben wir eine neue Themenreihe entworfen, in der aktuelle Aspekte der Thoraxonkologie diskutiert werden sollen, die folgende Themenbereiche umfassen:

- Welche relevanten prädiktiven Faktoren zur Therapieauswahl und -steuerung gibt es?

- Welche Testverfahren sind etabliert, und welche Testverfahren können wir in der Zukunft erwarten?

- Welche neuen Therapieansätze sind gegenwärtig in der Entwicklung?

- Welche Therapieoptionen gibt es bei Krankheitsprogress unter einer „targeted“ Therapie?

- Was ist die optimale Therapiesequenz in der Chemotherapie?
Eine Beantwortung dieser Fragestellungen im klinischen Alltag erfordert eine enge interdisziplinäre Zusammenarbeit, die nicht nur die verschiedenen ärztlichen Fachbereiche, sondern auch den Dialog zwischen niedergelassenen und im Krankenhaus tätigen Kollegen mit einschließt. Diese interdisziplinäre Betrachtungsweise soll auch in den jeweiligen Artikeln reflektiert werden.

In Zusammenarbeit mit Herrn M. Reck, A. Warth und A. Gröschel und R. Wiewrodt wurden bereits verschiedene Themenbereiche definiert. Als erstes Thema werden in dieser Ausgabe neue Ergebnisse zum Wirkstoff Crizotinib dargestellt, welcher jüngst zur Behandlung des ALK-positiven nicht-kleinzelligen Lungenkarzinoms (NSCLC) zugelassen wurde. Nachfolgend sind Übersichten zum EGFR-Signalweg, zur neuen histologischen Einteilung von pulmonalen Adenokarzinomen, zu aktuellen Konzepten und Ergebnissen der Immuntherapie beim NSCLC sowie Artikel zur Antiangiogenese und Zielstrukturen zur Behandlung des squamösen NSCLC vorgesehen.

Wir freuen uns auf Ihr Interesse an dieser neuen Übersichts-Reihe „Neue Wege in der ThoraxOnkologie“ und auf Ihre Vorschläge für weitere mögliche Themen und Autoren. 